\title{
Oxygen cost of dynamic leg exercise on a cycle ergometer: effects of gravity acceleration
}

\author{
M. GIRARDIS,${ }^{1}$ D. LINNARSSON,${ }^{2}$ C. MOIA, ${ }^{1}$ D.R. PENDERGAST ${ }^{3}$ and \\ G. FERRETTI ${ }^{1}$ \\ 1 Département de Physiologie, Centre Médical Universitaire, 1 rue Michel Servet, 1211 Genève, Switzerland \\ 2 Environmental Physiology Laboratory, Department of Physiology and Pharmacology, Karolinska Institute, Stockholm, Sweden \\ 3 Department of Physiology, State University of New York at Buffalo, Buffalo, New York, USA
}

\begin{abstract}
A model of the metabolic internal power $\left(\dot{E}_{\text {int }}\right)$ during cycling, which includes the gravity acceleration $\left(a_{\mathrm{g}}\right)$ as a variable, is presented. This model predicts that $\dot{E}_{\text {int }}$ is minimal in microgravity $(0 \mathrm{~g}$; $\left.g=9.81 \mathrm{~m} \mathrm{~s}^{-2}\right)$, and increases linearly with $a_{\mathrm{g}}$, whence the hypothesis that the oxygen uptake $\left(\dot{V}_{\mathrm{O}_{2}}\right)$ during cycling depends on $a_{g}$. Repeated $\dot{V}_{\mathrm{O}_{2}}$ measurements during steady-state exercise at 50,75 and $100 \mathrm{~W}$ on the cycle ergometer, performed in space $(0 \mathrm{~g})$ and on Earth $(1 \mathrm{~g})$ on two subjects, validated the model. $\dot{V}_{\mathrm{O}_{2}}$ was determined from the time course of decreasing $\mathrm{O}_{2}$ fraction during rebreathing. The gas volume during rebreathing was determined by the dilution principle, using an insoluble inert gas $\left(\mathrm{SF}_{6}\right)$. Average $\dot{V}_{2}$ for subject 1 at each power was $0.99,1.21$ and $1.52 \mathrm{~L} \mathrm{~min}^{-1}$ at $1 \mathrm{~g}(n=3)$ and $0.91,1.13$ and $1.32 \mathrm{~L} \mathrm{~min}^{-1}$ at $0 \mathrm{~g}(n=5)$. For subject 2 it was $0.90,1.12$ and $1.42 \mathrm{~L} \mathrm{~min}^{-1}$ at $1 \mathrm{~g}$, and $0.76,0.98$ and $1.21 \mathrm{~L} \mathrm{~min}^{-1}$ at $0 \mathrm{~g}$. These values corresponded to those predicted from the model. Although resting $\dot{V}_{\mathrm{O}_{2}}$ was lower at $0 \mathrm{~g}$ than at $1 \mathrm{~g}$, the net (total minus resting) exercise $\dot{V}_{\mathrm{O}_{2}}$ was still smaller at $0 \mathrm{~g}$ than at $1 \mathrm{~g}$. This difference reflects the lower $\dot{E}_{\text {int }}$ at $0 \mathrm{~g}$.
\end{abstract}

Keywords exercise, gravity acceleration, humans, internal work, oxygen uptake.

Received 14 December 1998, accepted 19 April 1999

The overall metabolic power developed by humans during dynamic leg exercise on a cycle ergometer is the sum of at least two components: (i) external power, or the power required to overcome the resistance applied to the ergometer, (ii) internal power, or the rate of energy expenditure required to move the lower limbs without opposition against an external load. The metabolic external power at any given pedalling frequency $\left(f_{\mathrm{p}}\right)$ is a linear function of mechanical power, and thus of the resistance applied to the ergometer (Dickinson 1929, Garry \& Wishart 1931, Henry \& De Moor 1950, Banister \& Jackson 1967, Whipp \& Wasserman 1969, Gaesser \& Brooks 1975). The reciprocal of the slope of this relationship has been defined as the delta-efficiency of exercise (Gaesser \& Brooks 1975).

The relationship between metabolic power and mechanical power takes different $y$-intercepts when weights are added to the legs (Kamon et al. 1973) or gravity acceleration $\left(a_{\mathrm{g}}\right)$ is increased (Bjurstedt et al. 1968). Cotes (1969) was the first to attribute these differences to changes in the cost of lifting the mass of the legs during pedalling. This qualitative statement introduced the concept of internal power during cycling, which became the object of several investigations (Kaneko \& Yamazaki 1978, Wells et al. 1986, Luhtanen et al. 1987, Widrick et al. 1992). The $f_{\mathrm{p}}$ and the mass of the legs $\left(m_{\mathrm{L}}\right)$ were identified as two of the determinants of internal power (Wells et al. 1986, Widrick et al. 1992, Francescato et al. 1995).

In this paper we propose a more complete model of metabolic internal power during cycling, which adds $a_{\mathrm{g}}$ to $f_{\mathrm{p}}$ and $m_{\mathrm{L}}$ as an independent determinant of it. In fact, owing to asymmetries in the pedalling cycle, the rotational movement of the legs is opposed, besides the external load, by the gravitational force and by inertial forces. Inertia accounts for a small fraction of the overall acceleration, and can be neglected in the present context. Gravity acceleration however, cannot be ignored as a determinant of internal work, although it was in previous studies, probably because it was considered invariant on Earth. 
The present model (see Theory) predicts that the metabolic internal power increases linearly with $a_{\mathrm{g}}$, and is minimal in microgravity. This being the case, the rate of oxygen uptake $\left(\dot{V} \mathrm{O}_{2}\right)$ during cycling against a given external load would partly depend on gravity acceleration. Lower $\dot{V}_{\mathrm{O}_{2}}$ values would be expected at the steady-state of given submaximal powers in microgravity $\left(0 \mathrm{~g} ; g=9.81 \mathrm{~m} \mathrm{~s}^{-2}\right)$ than on Earth $(1 \mathrm{~g})$.

Validation of this model was performed by means of $\dot{V} \mathrm{O}_{2}$ values obtained during dynamic leg exercise on the cycle ergometer at various submaximal powers (i) on Earth $(1 g)$ vs. a long-term space flight $(0 g)$, and (ii) on Earth vs. the human centrifuge at $3 \mathrm{~g}$. The former data are new and unpublished, so they are presented in detail in the Methods and Results sections. The latter data are from the literature (Bjurstedt et al. 1968, Rosenhamer 1968, Nunneley \& Shindell 1975), and are introduced in Discussion.

\section{THEORY}

During exercise at submaximal powers below the socalled lactate threshold, the net (total minus resting) $\dot{V} \mathrm{O}_{2}$ of the whole body is equivalent to the net rate of energy expenditure $(\dot{E}) . \dot{E}$ is equal to the sum of the rates of energy expenditure required to overcome external $\left(\dot{E}_{\text {ext }}\right)$ and internal $\left(\dot{E}_{\text {int }}\right)$ powers. $\dot{E}_{\text {ext }}$ is a linear function of the external mechanical power applied on the ergometer $(\dot{w})$, as follows:

$\dot{E}_{\text {ext }}=\dot{w} \Delta \eta^{-1}$

where the constant $\Delta \eta$, that is the reciprocal of the slope of this relationship, is the delta-efficiency of dynamic leg exercise (Gaesser \& Brooks 1975). This yields:

$\dot{E}=\dot{w} \Delta \eta^{-1}+\dot{E}_{\text {int }}$

$\dot{E}_{\text {int }}$ is independent of $\dot{w}$, is a power function of the pedalling frequency $\left(f_{\mathrm{p}}\right)$ (di Prampero et al. 1979) and is a linear function of the weight of the legs, that is the product of the mass of the legs $\left(m_{\mathrm{L}}\right)$ times $a_{\mathrm{g}}$. For a given $f_{\mathrm{p}}$ this implies:

$\dot{E}_{\text {int }}=\varepsilon m_{\mathrm{L}} a_{\mathrm{g}}$

where the constant $\varepsilon$, which has the dimension of a velocity, contains the length of the pedal lever and the fourth power of $f_{\mathrm{p}}$. The equation relating $\varepsilon$ to $f_{\mathrm{p}}$ has been derived, by introducing $a_{\mathrm{g}}$ and correcting for appropriate units, from the empirical data of Francescato et al. (1995), obtained over an $f_{\mathrm{p}}$ range from 40 to $100 \mathrm{~min}^{-1}$ :

$\varepsilon=0.112 f_{\mathrm{P}}^{4.165}$

where $\varepsilon$ is in $\mathrm{m} \mathrm{s}^{-1}$ and $f_{\mathrm{p}}$ in $\mathrm{s}^{-1}$. Substitution of Eqn 4 in Eqn 3 yields:
$\dot{E}_{\text {int }}=0.112 m_{\mathrm{L}} a_{\mathrm{g}} f_{\mathrm{P}}^{4.165}$

where $\dot{E}_{\text {int }}$ is in $\mathrm{W}, m_{\mathrm{L}}$ is in $\mathrm{kg}$ and $a_{\mathrm{g}}$ is in $\mathrm{m} \mathrm{s}^{-2}$. Francescato et al. described also a quadratic equation relating $\Delta \eta$ to $f_{\mathrm{p}}$ during cycling, as follows:

$\Delta \eta=0.13 f_{\mathrm{P}}^{2}-0.18 f_{\mathrm{P}}+0.326$

Substitution of Eqns 5 and 6 in Eqn 2 yields:

$E=\dot{w}\left(0.13 f_{\mathrm{P}}^{2}-0.18 f_{\mathrm{P}}+0.326\right)^{-1}+0.112 m_{\mathrm{L}} a_{\mathrm{g}} f_{\mathrm{P}}^{4.165}$

Eqn 7 describes the overall relationship between $\dot{E}, m_{\mathrm{L}}$, $a_{\mathrm{g}}, f_{\mathrm{p}}$ and $\dot{w}$ during exercise on the cycle ergometer. It is noteworthy that at $0 \mathrm{~g}$, the second term of the righthand branch of Eqn 7, designating $\dot{E}_{\text {int }}$, equals 0 , so that $\dot{E}$ is equal to $\dot{E}_{\text {ext }}$, and therefore varies only as a function of $w$ and $f_{\mathrm{p}}$.

It follows from Eqn 7 that, at any given power output below the so-called lactate threshold, the oxygen uptake at the exercise steady-state $\left(\dot{V}_{\mathrm{O}_{2}}\right)$ varies with $a_{\mathrm{g}}$, being minimal at $0 \mathrm{~g}$ (space flight) irrespective of the $m_{\mathrm{L}}$, and being increased in hypergravity environments, as can be obtained in the human centrifuge, with respect to the values usually observed on Earth, at $1 \mathrm{~g}$.

It is noteworthy that the internal power includes also a component related to frictional and visco-elastic forces developing inside the contracting muscles. This component cannot be quantified, is likely independent of $a_{\mathrm{g}}$ and is not taken into account as such in this model. Yet it is measured with $\dot{E}$ and is likely, although in formally inappropriate manner, concealed in the first term of the right-hand branch of Eqn 7, as it is expected to vary with $\dot{w}$ and $f_{\mathrm{p}}$.

\section{METHODS}

\section{Subjects}

A total of 10 determinations of steady-state $\dot{V} \mathrm{O}_{2}$ during submaximal dynamic leg exercise were obtained on two subjects (age 37 years and body mass before flight $75.1 \mathrm{~kg}$ for subject 1 ; age 39 years and body mass before flight $68.9 \mathrm{~kg}$ for subject 2), who flew onboard the Russian Space Station Mir for 6 months. Both subjects were perfectly aware of the procedures and of their implicit risks, and gave their informed consent. Ethical and medical approval to the experiments was given by the Medical Board of the European Space Agency.

The $m_{\mathrm{L}}$ was not measured, and it was assumed to be $32.2 \%$ of total body mass on Earth (Winter 1979). This estimate was used for the data obtained at $1 \mathrm{~g}$, prior to the space flight. No attempt of estimating $m_{\mathrm{L}}$ at $0 \mathrm{~g}$ was made, because, according to Eqn 5 , the $\dot{E}_{\text {int }}$ at $0 g$ is by definition equal to $0 \mathrm{~W}$, irrespective of $m_{\mathrm{L}}$. 


\section{Measurements and calculations}

The $\dot{V} \mathrm{O}_{2}$ was measured by a closed circuit method during rebreathing. The rebreathing bag was filled with a mixture containing $\mathrm{O}_{2}$, a soluble inert gas $\left(\mathrm{N}_{2} \mathrm{O}\right.$, added for other purposes) and an insoluble inert gas $\left(\mathrm{SF}_{6}\right)$, balanced with $\mathrm{N}_{2}$. This mixture was diluted with ambient air using a calibrated syringe, to spare gas during the flight. The initial $\mathrm{O}_{2}$ fraction in the bag ranged between 0.326 and 0.375 , in order to avoid the occurrence of hypoxia at the end of the rebreathing manoeuvre. The volume of the rebreathing bag was empirically established prior to the first experiment, it was increased with the power output and ranged between 2.5 and $3.5 \mathrm{~L}$. During rebreathing, the dry fractions of $\mathrm{O}_{2}, \mathrm{CO}_{2}$ and $\mathrm{SF}_{6}\left(F_{\mathrm{O}_{2}}, F_{\mathrm{CO}_{2}}\right.$ and $F \mathrm{SF}_{6}$, respectively) at the mouth were continuously monitored by a photo-acoustic gas analyser (RMS-II, Innovision A/S, Odense, Denmark), stored on a magnetic disk and subsequently analysed by means of Matlab software (Mathworks, USA). The combined accuracy of the gas delivery system and the $\mathrm{SF}_{6}$ analysis for determining gas volumes by dilution was checked repeatedly before and during the flight by adding known volumes of air to the rebreathing bag with the calibration syringe.

The knowledge of bag volume $\left(V_{\mathrm{bag}} ; \mathrm{L}\right)$ and initial bag $F_{\mathrm{SF}_{6}}\left[F_{\mathrm{SF}_{6}}\left(t_{0}\right)\right]$ allowed computation of the overall closed circuit volume (i.e. bag volume + dead space volume + alveolar volume, $V_{\text {sys }}$ ) from the time course of $F_{\mathrm{SF}_{6}}\left[\mathrm{FSF}_{6}(t)\right.$; from the third breath onward] using the dilution principle:

$$
V_{\text {sys }}(t)=\left[F_{\mathrm{SF}_{6}}\left(t_{0}\right) V_{\text {bag }}\right] F_{\mathrm{SF}_{6}}(t)^{-1}
$$

The amount of oxygen in the system at any breath $\left(V_{\mathrm{O}_{2}}(t)\right.$; (L) was obtained by multiplying $V_{\text {sys }}(t)$ times the corresponding end tidal oxygen fraction $\left[F_{\mathrm{et}} \mathrm{O}_{2}(t)\right]$. $V_{\mathrm{O}_{2}}(t)$ is linearly related to the rebreathing time $\left(t_{\mathrm{R}} ; \mathrm{min}\right)$, the slope being equal to $\dot{V}_{\mathrm{O}_{2}}$ : this allows computation of $\dot{V} \mathrm{O}_{2}\left(\mathrm{~L} \mathrm{~min}^{-1}\right)$ by the least mean square method. Fourto-seven breaths, at the end of which the $\mathrm{FO}_{2}$ inside the bag ranged between 0.246 and 0.193 , could be used for the computation of $\dot{V} \mathrm{O}_{2}$. The regression lines with a correlation coefficient below 0.95 were discarded. In all experiments, three rebreathing manoeuvres were performed at the exercise steady-state. Application of this procedure provided at least two valid $\dot{V} \mathrm{O}_{2}$ measurements at each power output, which were then averaged.

The heart rate was measured continuously by electrocardiography. As it did not increase significantly (mean increase $=9.7 \pm 4.6 \%$ ) during rebreathing at rest, correction of $\dot{V} \mathrm{O}_{2}$ for heart rate changes, as suggested by Kallay et al. (1990), was not performed.

The steady-state gas exchange ratio $(R)$ was calculated from the time course of $\mathrm{FO}_{2}$ and $\mathrm{FCO}_{2}$ at the mouth during regular breathing, by means of the alve- olar gas equations (Otis 1964). To this purpose, the $\mathrm{O}_{2}$ and $\mathrm{CO}_{2}$ traces were recorded between the second and the third rebreathing manoeuvres. End tidal gas fractions were assumed to be equivalent to alveolar gas fractions. The average steady-state $\mathrm{CO}_{2}$ output $\left(\dot{V} \mathrm{CO}_{2}\right)$ was calculated by multiplying $R$ times the corresponding average value for $\dot{V} \mathrm{O}_{2}$.

\section{Experimental design}

$\dot{V} \mathrm{O}_{2}, R$ and $\dot{V} \mathrm{CO}_{2}$ were determined at rest and during steady-state exercise requiring 50,75 and $100 \mathrm{~W}$ power outputs performed on an electrically braked cycle ergometer (Innovision A/S, Odense, Denmark). The subjects were requested to pedal at an $f_{\mathrm{p}}$ of $60 \mathrm{~min}^{-1}$. The ergometer used during the space flight was identical to that used on Earth. Both were calibrated by the manufacturer prior to the study and were shown to maintain their calibrated power levels for longer than one year. At each power output, rebreathing was performed at 3, 9 and 12 min of exercise. Breath-by-breath $\mathrm{O}_{2}$ and $\mathrm{CO}_{2}$ fractions were obtained between the first and the second rebreathing. Experiments at $1 \mathrm{~g}$ were carried out 172, 116 and 73 days before the flight on both subjects. The same protocol was repeated during a 180-day flight on board the Russian Space Station Mir $(0 \mathrm{~g}$, cabin temperature during the flight ranging between 20.6 and $24.5^{\circ} \mathrm{C}$, barometric pressure ranging between 710 and $788 \mathrm{mmHg}$ ) on days: 12, 54, 80, 122, 144 and 13, 59, 81, 117,143 after the launch on subject 1 and 2, respectively. Thus, for each power output, a total of six and ten observations could be performed at $1 \mathrm{~g}$ and $0 \mathrm{~g}$, respectively.

\section{Statistical analysis}

Comparison between data at $1 \mathrm{~g}$ and at $0 \mathrm{~g}$ was performed by the one-tailed unpaired $t$-test. Comparison of the measured $\dot{V} \mathrm{O}_{2}$ with the corresponding values predicted from the model was performed by means of onetailed paired $t$-test $(n=48)$. The relationship between $\dot{V} \mathrm{O}_{2}$ and power was determined by regression analysis and the comparison between regression lines was performed according to Kleinbaum et al. (1988). The comparison of the slope of a regression line with the slope of the identity line was performed as described by Kleinbaum et al. (1988), testing the hypothesis $\mathrm{H}_{0}$ : $\beta_{1}=1$. Data were considered significantly different when $P<0.05$.

\section{RESULTS}

The individual values of $\dot{V} \mathrm{O}_{2}$ and $\dot{V} \mathrm{CO}_{2}$ are reported in Table 1 . The $\dot{V} \mathrm{O}_{2}$ rates at $0 \mathrm{~g}$ appear not to vary with the time of flight. At $0 \mathrm{~g}$, lower $\dot{V} \mathrm{O}_{2}$ values were found for subject 2 than for subject 1, mostly because of lower resting $\dot{V} \mathrm{O}_{2}$ in the former. For both subjects, the $\dot{V} \mathrm{O}_{2} \mathrm{~S}$ 
Table 1 Oxygen uptake and carbon dioxide output for the two subjects before and during the space-flight

\begin{tabular}{|c|c|c|c|c|c|c|c|c|c|c|c|c|c|c|c|c|}
\hline & \multicolumn{4}{|l|}{ Rest } & \multicolumn{4}{|l|}{$50 \mathrm{~W}$} & \multicolumn{4}{|l|}{$75 \mathrm{~W}$} & \multicolumn{4}{|c|}{$100 \mathrm{~W}$} \\
\hline & \multicolumn{2}{|c|}{ Subject 1} & \multicolumn{2}{|c|}{ Subject 2} & \multicolumn{2}{|c|}{ Subject 1} & \multicolumn{2}{|c|}{ Subject 2} & \multicolumn{2}{|c|}{ Subject 1} & \multicolumn{2}{|c|}{ Subject 2} & \multicolumn{2}{|c|}{ Subject 1} & \multicolumn{2}{|c|}{ Subject 2} \\
\hline & $\dot{V} \mathrm{O}_{2}$ & $\dot{V} \mathrm{CO}_{2}$ & $\dot{V} \mathrm{O}_{2}$ & $\dot{V} \mathrm{CO}_{2}$ & $\dot{V}_{\mathrm{O}_{2}}$ & $\dot{V} \mathrm{CO}_{2}$ & $\dot{V} \mathrm{O}_{2}$ & $\dot{V} \mathrm{CO}_{2}$ & $\dot{V}_{\mathrm{O}_{2}}$ & $\dot{V} \mathrm{CO}_{2}$ & $\dot{V}_{\mathrm{O}_{2}}$ & $\dot{V} \mathrm{CO}_{2}$ & $\dot{V} \mathrm{O}_{2}$ & $\dot{V} \mathrm{CO}_{2}$ & $\dot{V}_{\mathrm{O}_{2}}$ & $\dot{V}_{\mathrm{CO}}$ \\
\hline \multicolumn{17}{|l|}{ Pre-flight day } \\
\hline$-172 /-172$ & 0.46 & 0.39 & 0.24 & 0.22 & 0.96 & 0.88 & 0.89 & 0.87 & 1.23 & 1.07 & 1.07 & 1.14 & 1.58 & 1.46 & 1.36 & 1.23 \\
\hline$-116 /-116$ & 0.34 & 0.34 & 0.28 & 0.26 & 1.04 & 1.06 & 0.94 & 0.88 & 1.30 & 1.31 & 1.12 & 1.11 & 1.56 & 1.58 & 1.52 & 1.55 \\
\hline$-73 /-73$ & 0.36 & 0.30 & 0.31 & 0.25 & 0.95 & 0.84 & 0.87 & 0.77 & 1.09 & 0.94 & 1.16 & 1.03 & 1.53 & 1.35 & 1.37 & 1.27 \\
\hline Mean & 0.39 & 0.35 & 0.28 & 0.24 & 0.99 & 0.93 & 0.90 & 0.84 & 1.21 & 1.11 & 1.12 & 1.09 & 1.52 & 1.43 & 1.42 & 1.35 \\
\hline $\mathrm{SD}$ & 0.06 & 0.04 & 0.06 & 0.02 & 0.05 & 0.12 & 0.03 & 0.06 & 0.11 & 0.19 & 0.04 & 0.06 & 0.04 & 0.14 & 0.09 & 0.17 \\
\hline Predicted $\dot{V} \mathrm{O}_{2}$ & & & & & 0.99 & & 0.87 & & 1.25 & & 1.13 & & 1.51 & & 1.39 & \\
\hline \multicolumn{17}{|l|}{ Flight day } \\
\hline$+12 /+13$ & 0.27 & 0.30 & 0.22 & 0.21 & 0.86 & 0.73 & 0.75 & 0.63 & 1.04 & 0.89 & 0.97 & 0.84 & 1.35 & 1.24 & 1.23 & 1.16 \\
\hline$+54 /+59$ & 0.31 & 0.26 & 0.21 & 0.17 & 0.96 & 0.80 & 0.79 & 0.75 & 1.14 & 0.92 & 0.98 & 0.91 & 1.26 & 1.08 & 1.12 & 1.08 \\
\hline$+80 /+81$ & 0.33 & 0.28 & 0.19 & 0.15 & 0.90 & 0.71 & 0.67 & 0.60 & 1.16 & 1.10 & 0.90 & 0.83 & 1.31 & 1.10 & 1.19 & 1.11 \\
\hline$+122 /+117$ & 0.33 & 0.29 & 0.25 & 0.22 & 0.91 & 0.78 & 0.83 & 0.74 & 1.14 & 1.02 & 1.02 & 0.97 & 1.39 & 1.26 & 1.28 & 1.16 \\
\hline$+144 /+143$ & 0.33 & 0.30 & 0.24 & 0.19 & 0.94 & 0.89 & 0.77 & 0.66 & 1.16 & 1.15 & 1.02 & 0.89 & 1.28 & 1.13 & 1.24 & 1.10 \\
\hline Mean & 0.31 & 0.29 & 0.22 & 0.19 & 0.91 & 0.78 & 0.76 & 0.67 & 1.13 & 1.02 & 0.98 & 0.89 & 1.32 & 1.16 & 1.21 & 1.12 \\
\hline $\mathrm{SD}$ & 0.03 & 0.02 & 0.02 & 0.03 & 0.04 & 0.07 & 0.06 & 0.07 & 0.05 & 0.11 & 0.05 & 0.06 & 0.05 & 0.08 & 0.06 & 0.04 \\
\hline Predicted $\dot{V} \mathrm{O}_{2}$ & & & & & 0.83 & & 0.74 & & 1.09 & & 1.00 & & 1.35 & & 1.26 & \\
\hline
\end{tabular}

Day 0 is the day of launch. $\dot{V}_{\mathrm{O}_{2}}$ is oxygen uptake, $\dot{V}_{\mathrm{CO}_{2}}$ is carbon dioxide output, both given in $\mathrm{L} \mathrm{min}^{-1}$. SD is standard deviation. The reported $\dot{V}_{\mathrm{O}_{2}}$ values are measured gross $\dot{V}_{\mathrm{O}_{2}}$ values. Measured resting $\dot{V}_{\mathrm{O}_{2}}$ values and Eqn 8 have been used to compute the predicted $\dot{V}_{\mathrm{O}_{2}}$, reported in bold, for preflight $(1 \mathrm{~g})$ and flight $(0 \mathrm{~g})$.

at $0 \mathrm{~g}$ were significantly and systematically lower than at $1 \mathrm{~g}$ at all powers. The overall mean differences between the two conditions were $0.105 \mathrm{~L} \mathrm{~min}^{-1} \quad(n=92$; $P<0.05)$ and $0.138 \mathrm{~L} \mathrm{~min}^{-1}(n=92 ; P<0.05)$ for subject 1 and 2 , respectively. The differences in $\dot{V}_{\mathrm{O}_{2}}$ between 0 and $1 g$ during exercise were partly owing to a lower resting $\dot{V}_{\mathrm{O}_{2}}$ in the former than in the latter condition. However, also the net (total minus resting) $\dot{V}_{\mathrm{O}_{2}}$ values during exercise were smaller at $0 \mathrm{~g}$ than at $1 \mathrm{~g}$ (0.046 and $0.103 \mathrm{~L} \mathrm{~min}^{-1}$, average difference for subject 1 and 2, respectively), although this difference was not significant for subject 1 .

The $R$ was the same in both conditions $(0.92 \pm 0.07$ and $0.89 \pm 0.07$ for subject $1,0.93 \pm 0.07$ and $0.89 \pm 0.05$ for subject 2 , mean of all determinations at $1 \mathrm{~g}$ and $0 \mathrm{~g}$, respectively). As a consequence, $\dot{V} \mathrm{CO}_{2}$ turned out to be lower at $0 \mathrm{~g}$ than at $1 \mathrm{~g}$, the overall mean difference being $0.140 \mathrm{~L} \mathrm{~min}^{-1} \quad(n=32$; $P=0.09)$ and $0.163 \mathrm{~L} \mathrm{~min}^{-1}(n=32 ; P=0.08)$ for subject 1 and 2 , respectively.

The measured $\dot{V} \mathrm{O}_{2}$ is plotted in Fig. 1 against the corresponding $\dot{V} \mathrm{O}_{2}$ values predicted from Eqn 7 , after proper unit conversion. At $1 \mathrm{~g}$, the measured $\dot{V}_{\mathrm{O}_{2}}$ appears to be equal to the predicted $\dot{V}_{\mathrm{O}_{2}}$ $(y=1.013 x-0.005, R=0.945$, equal to the identity line). At $0 \mathrm{~g}$, a positive intercept is observed, the slope being lower than $1(y=0.819 x+0.146, R=0.968$, different from the identity line).

The linear increases of $\dot{V}_{\mathrm{O}_{2}}\left(\mathrm{~L} \mathrm{~min}^{-1}\right)$ as a function of mechanical power $(\dot{w} ; \mathrm{W})$ are shown in Fig. 2 for

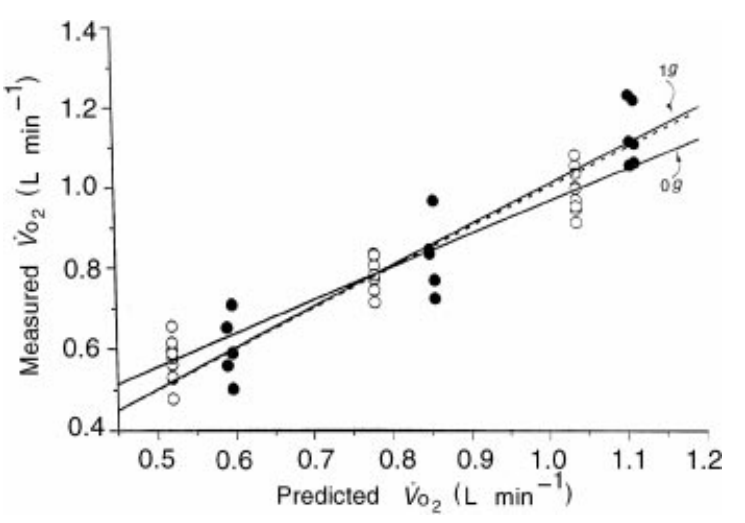

Figure 1 A comparison of measured and predicted values of oxygen uptake. Measured oxygen uptake is plotted as a function of predicted oxygen uptake $\left(\dot{V}_{\mathrm{O}}, \mathrm{L} \mathrm{min}^{-1}\right)$. The dashed line is the identity line. Continuous lines are the regression lines for $1 \mathrm{~g}\left(g=9.81 \mathrm{~m} \mathrm{~s}^{-2}\right)($ and $0 g(\bigcirc)$. Regression equations are: at $1 g, y=1.013 x-0.005$, $R=0.945 ;$ at $0 g, y=0.819 x+0.146, r=0.968$. The latter equation is significantly different from the identity line.

both 0 and $1 \mathrm{~g}$. Regression analysis indicates that: (i) the $y$-intercept at $0 g\left(0.349\right.$ and $\left.0.235 \mathrm{~L} \mathrm{~min}^{-1}\right)$ was lower than at $1 g\left(0.393\right.$ and $\left.0.295 \mathrm{~L} \mathrm{~min}^{-1}\right)$ and (ii) the slope at $0 \mathrm{~g}$ was smaller than at $1 \mathrm{~g}(P<0.05)$. The slope of this line is the reciprocal of the $\Delta \eta$ of dynamic leg exercise (Gaesser \& Brooks 1975). Thus $\Delta \eta$ was inevitably greater at $0 \mathrm{~g}$ than at $1 \mathrm{~g}(28.3$ and $29.0 \%$ at $0 \mathrm{~g}$ in subjects 1 and 2, respectively, to be compared with 25.6 and $25.4 \%$ at $1 \mathrm{~g}$, respectively). 

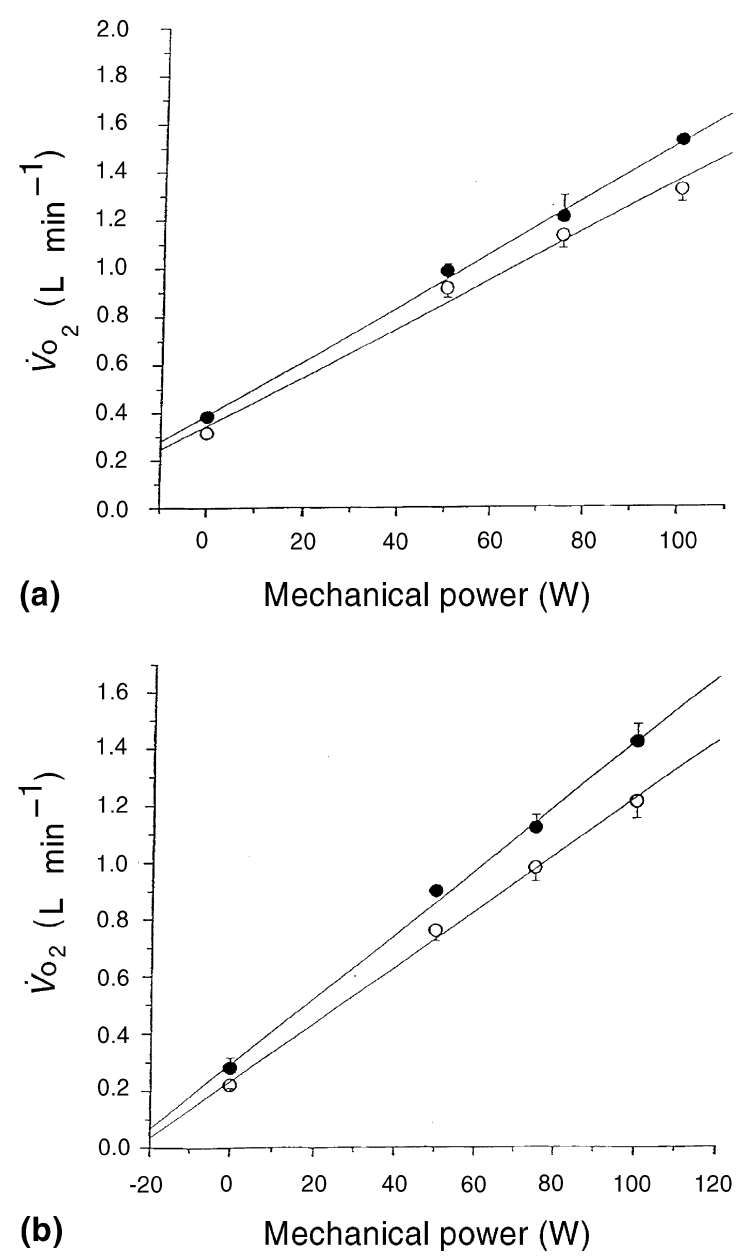

Figure 2 Oxygen uptake as a function of power. Oxygen uptake $\left(\dot{V}_{\mathrm{O}_{2}}, \mathrm{~L} \mathrm{~min}{ }^{-1}\right)$ as a function of external mechanical power (in $\mathrm{W}$ ), for subjects 1 (a) and 2 (b). Data are plotted as mean and standard deviation. Filled circles, data at $1 \mathrm{~g}\left(\mathrm{~g}=9.81 \mathrm{~m} \mathrm{~s}^{-2}\right)$; open circles, data at $0 \mathrm{~g}$. Regression lines at 1 and $0 \mathrm{~g}$ were calculated on individual $\dot{V} \mathrm{O}_{2}$ values.

The individual linear relationships between heart rate and $\dot{V} \mathrm{O}_{2}$ at 1 and $0 \mathrm{~g}$ are reported in Fig. 3. No significant differences between the two tested conditions were found for either subject. Extrapolation of the regression lines to the predicted maximal heart rates for the age of each subject allows an estimate of the individual maximal $\dot{V} \mathrm{O}_{2}$ at 1 and $0 \mathrm{~g}$. Estimated maximal $\dot{V} \mathrm{O}_{2}$ values were 3.11 and $2.87 \mathrm{~L} \mathrm{~min}^{-1}$ for subject 1 , and 2.83 and $2.80 \mathrm{~L} \mathrm{~min}^{-1}$ for subject 2 , at $1 \mathrm{~g}$ and $0 \mathrm{~g}$, respectively. The highest submaximal $\dot{V} \mathrm{O}_{2}$ values measured in this study correspond thus to about one half of the individual maximal $\dot{V} \mathrm{O}_{2}$.

\section{DISCUSSION}

The generalized model describing the factors that affect the rate of energy expenditure during dynamic leg exercise on the cycle ergometer appears to be a valid
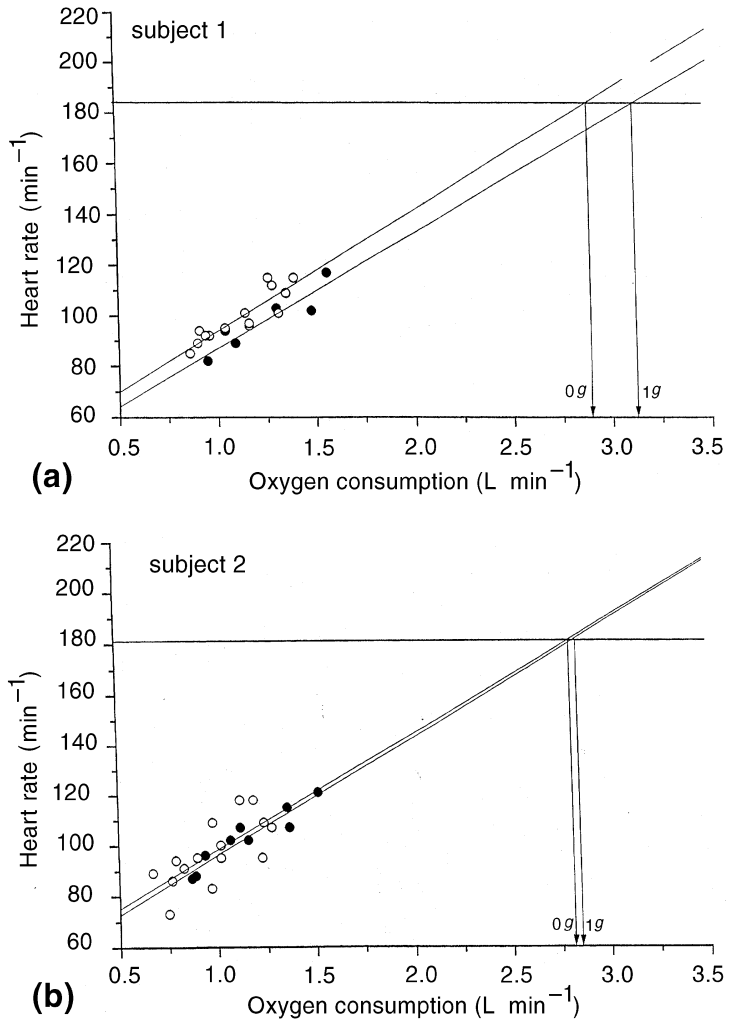

Figure 3 Estimate of maximal oxygen consumption at $0 \mathrm{~g}$. Heart rate as a function of oxygen consumption at $1 \mathrm{~g}(\mathbf{O})$ and $0 \mathrm{~g}(\bigcirc)$ for the two subjects who flew for 6 months in the Russian Space Station Mir.

predictor of $\dot{E_{\text {int }}}$ and of the $\dot{V} \mathrm{O}_{2}$ changes as a function of $a_{\mathrm{g}}$ at a given imposed external load. The $\dot{V} \mathrm{O}_{2}$ values measured in this study correspond to the theoretical values that were obtained from the model. The exception was subject 1 at $0 \mathrm{~g}$, probably because he tended to vary his $f_{\mathrm{p}}$ at higher powers, so that his data did not entirely fulfil the constraints of the model for $f_{\mathrm{p}}=60 \mathrm{~min}^{-1}$. Unfortunately, although the subjects were asked to pedal at $f_{\mathrm{p}}=60 \mathrm{~min}^{-1}, f_{\mathrm{p}}$ was not recorded by the operator: this needs to be acknowledged as a limitation of this study. Nevertheless, the hypothesis derived from the generalized model of $\dot{E}_{\text {int }}$, that the $\dot{V} \mathrm{O}_{2}$ during submaximal dynamic exercise on the cycle ergometer at $0 \mathrm{~g}$ would be lower than that at the same absolute power at $1 \mathrm{~g}$ is supported by the results.

The observation of lower resting $\dot{V} \mathrm{O}_{2}$ values at $0 \mathrm{~g}$ than at $1 \mathrm{~g}$ is consistent with a decrease in postural muscle contractions in the former condition (Prisk et al. 1995), but is at variance with the reported finding of a higher resting $\dot{V} \mathrm{O}_{2}$ from previous space missions (Michel et al. 1977). Clearly enough, this finding cannot entirely explain the observed decrease in $\dot{V} \mathrm{O}_{2}$ during exercise at $0 \mathrm{~g}$. In fact, in agreement with the data of Michel et al. (1977), net $\dot{V} \mathrm{O}_{2}\left(\dot{V} \mathrm{O}_{2}\right.$ minus resting $\left.\dot{V} \mathrm{O}_{2}\right)$ at $0 \mathrm{~g}$ is still lower than at $1 \mathrm{~g}$, the difference being significant in subject 2 . It has been proposed that the 
difference between net $\dot{V} \mathrm{O}_{2}$ at 1 and at $0 \mathrm{~g}$ be owing to an increase in the time constant of the $\dot{V} \mathrm{O}_{2}$ kinetics caused by microgravity adaptation (Convertino 1996). The results of the present study do not support this hypothesis. If the $\dot{V} \mathrm{O}_{2}$ kinetics were slow enough to affect the measured $\dot{V} \mathrm{O}_{2}$, the $\dot{V} \mathrm{O}_{2}$ values obtained in the three subsequent rebreathing manoeuvres (at $\mathrm{min} 3$, 9 and 12 of exercise) would have increased progressively, which was not the case.

The lower net $\dot{V} \mathrm{O}_{2}$ during submaximal exercise on the cycle ergometer at any given power output at $0 \mathrm{~g}$ than at $1 \mathrm{~g}$ is rather a consequence of a lower $\dot{E}_{\text {int }}$ at $0 \mathrm{~g}$. In fact Eqns 3 and 5 show that $\dot{E}_{\text {int }}$ : (i) is a linear function of $a_{\mathrm{g}}$, (ii) is independent of the external mechanical power, and (iii) at $0 \mathrm{~g}$ is equal to $0 \mathrm{~W}$, which implies that it is not affected by the changes in $m_{\mathrm{L}}$ that occur during microgravity exposure (Le Blanc et al. 1995). Incidentally, it is noteworthy that $\dot{E}_{\text {int }}$ is directly proportional to $m_{\mathrm{L}}$, which may explain why obese subjects have a higher oxygen cost of cycling at a given work rate than lean individuals (Åstrand et al. 1960, Anton-Kuchly et al. 1984). At $0 \mathrm{~g}{\dot{E_{\text {int }}}}=0 \mathrm{~W}$, the difference between the $y$-intercept of the linear relationship between $\dot{V} \mathrm{O}_{2}$ and $\dot{w}$ (Fig. 2) at $1 \mathrm{~g}$ and the $y$-intercept of the same relationship at $0 \mathrm{~g}$ includes the differences in resting $\dot{V}_{\mathrm{O}_{2}}$ plus the $\dot{E}_{\text {int }}$ at $1 \mathrm{~g}$. Such a difference for subject 2 is remarkably close to the $\dot{E}_{\text {int }}$ values that can be estimated from Eqn 5, after correcting for the decreased resting $\dot{V} \mathrm{O}_{2}$ at $0 \mathrm{~g}$.

If indeed $\dot{E}_{\text {int }}$ includes all the gravity-dependent components of internal work during dynamic leg exercise on the cycle ergometer, the $\dot{V} \mathrm{O}_{2}$ vs. power lines at $1 \mathrm{~g}$ and $0 \mathrm{~g}$ ought to be parallel, the difference between them being equal to $\dot{E}_{\text {int }}$. However, this is not the case, because the difference between net $\dot{V} \mathrm{O}_{2}$ at 1 and $0 \mathrm{~g}\left(\dot{V}_{\mathrm{O}_{2}}, 1 \mathrm{~g}-\dot{V} \mathrm{O}_{2}, 0 \mathrm{~g} ; \mathrm{mL} \mathrm{O}_{2} \mathrm{~min}^{-1}\right)$ becomes progressively greater as external power is increased (on average 35,40 and $135 \mathrm{~mL} \mathrm{O}_{2} \mathrm{~min}^{-1}$ at 50, 75 and $100 \mathrm{~W}$, respectively), and the slopes of the lines relating $\dot{V} \mathrm{O}_{2}$ to external power, shown in Fig. 2, are lower at $0 \mathrm{~g}$ than at $1 \mathrm{~g}$ (greater $\Delta \eta$ ). The discrepancy between $\dot{E}_{\text {int }}$ and $\dot{V} \mathrm{O}_{2}, 1 g-\dot{V} \mathrm{O}_{2}, 0 g$ might be caused by changes in $f_{\mathrm{p}}$. This would also explain the observation that the relationship between measured and predicted $\dot{V} \mathrm{O}_{2}$ has a slope lower than 1 and a significant positive $y$-intercept (see Fig. 1). In fact subject 1 reportedly tended to increase his $f_{\mathrm{p}}$ at increasing power outputs at $0 \mathrm{~g}$.

A progressively greater respiratory work at increasing external powers is a possible alternative explanation of this finding. Indeed, the $\dot{V} \mathrm{O}_{2}, 1 \mathrm{~g}-\dot{V} \mathrm{O}_{2}, 0 \mathrm{~g}$ difference appears compatible with known values of respiratory work during exercise in humans (Cerretelli \& di Prampero 1987). However, the hypothesis that respiratory work explains the entire difference between $\dot{V}_{\mathrm{O}_{2}}, 1 g-\dot{V} \mathrm{O}_{2}, 0 g$ and $\dot{E}_{\text {int }}$ implies that the respi- ratory work at $0 \mathrm{~g}$ be lower than at $1 \mathrm{~g}$ at any power output. To our knowledge no data were reported on respiratory work during space flight. Further investigations need to be carried out on this subject.

At higher powers than the so-called lactate threshold, a steady $\dot{V} \mathrm{O}_{2}$ level is not always attained, but a slow and continuous increase in $\dot{V} \mathrm{O}_{2}$ occurs (Whipp \& Wasserman 1972, Linnarsson 1974, Camus et al. 1988). The comparison between measured and theoretical $\dot{V} \mathrm{O}_{2}$ values, as performed in this study, is valid only as far as the exercise $\dot{V} \mathrm{O}_{2}$ is obtained at external powers below the lactate threshold. This was probably the case for both subjects, as their gas exchange ratio is below 1 , and low heart rate values were attained even at the highest $\dot{w}$. Extrapolation of the heart rate vs. $\dot{V} \mathrm{O}_{2}$ relationship to the predicted individual maximum suggests that (i) the highest investigated $\dot{w}$ was approximately one half of the estimated maximum, and (ii) the predicted maximal $\dot{V} \mathrm{O}_{2}$ at $0 \mathrm{~g}$ was similar to that at $1 \mathrm{~g}$. The latter estimate agrees with the few data reported in the literature, showing that the maximal $\dot{V} \mathrm{O}_{2}$ at $0 \mathrm{~g}$ is either increased after a long-term space flight with an intense training regimen (Michel et al. 1977) or unchanged after short-term space flight (Levine et al. 1996). As any given $\dot{V} \mathrm{O}_{2}$ level is attained at a higher $\dot{w}$ at $0 \mathrm{~g}$ than at $1 \mathrm{~g}$, unchanged maximal $\dot{V} \mathrm{O}_{2}$ values imply higher maximal mechanical powers in the former than in the latter condition. Thus, the maximal working capacity should be greater in microgravity than on Earth. The opposite would occur on planets with masses greater than that of the Earth, and not only because of the upward displacement of the $\dot{V} \mathrm{O}_{2}$ vs. $\dot{w}$ relationship at $1 \mathrm{~g}$, but also because the maximal $\dot{V} \mathrm{O}_{2}$ decreases in the latter condition (Pendergast et al. 1987). Because of exponent 4 for $f_{\mathrm{p}}$ in Eqns 4 and 5, the variations of maximal aerobic power as a function of $a_{\mathrm{g}}$ would become greater if the frequency of movement is increased.

Application of Eqn 7 allows a comparison of the present results with the data obtained at higher $g$-values in the human centrifuge. During exercise on a cycle ergometer in the centrifuge the difference between $\dot{V} \mathrm{O}_{2}$ at 3 and $1 \mathrm{~g}$ (Bjurstedt et al. 1968, Rosenhamer 1968, Nunneley \& Shindell 1975) appears to be very close to that expected from Eqn 3. For instance, Bjurstedt et al. (1968) reported a mean difference (three different power outputs) between net $\dot{V} \mathrm{O}_{2}$ at 3 and $1 \mathrm{~g}$ of about $0.137 \mathrm{~L} \mathrm{~min}^{-1}$ which is quite similar to that calculated from Eqn $3\left(0.134 \mathrm{~L} \mathrm{~min}^{-1}\right)$ after the insertion of appropriate data $\left(f_{\mathrm{p}}=60\right.$ r.p.m., $m_{\mathrm{L}}=32.2 \%$ of body weight $=21.4 \mathrm{~kg}_{\mathrm{m}}$ ). Bjurstedt et al. (1968) attributed this difference to an increased work of respiratory and postural muscles at $3 \mathrm{~g}$. This hypothesis was questioned by Nunneley \& Shindell (1975) who suggested that the observed $\dot{V} \mathrm{O}_{2}$ difference was too large to be explained by increased respiratory and 


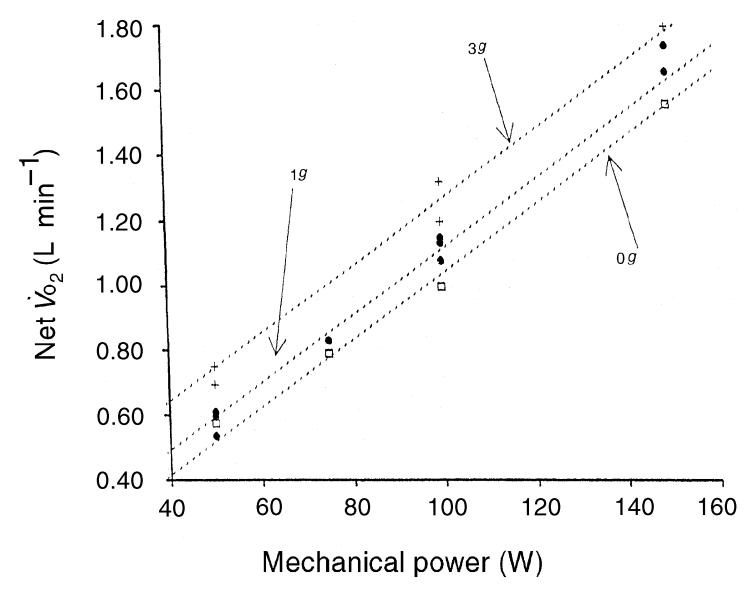

Figure 4 Effect of gravity acceleration on oxygen uptake during exercise. Net oxygen uptake (net $\dot{V} \mathrm{O}_{2}$ ) as a function of mechanical power as from Eqn 8 at varying gravity acceleration $(0,1,3 \mathrm{~g}$; $\left.g=9.81 \mathrm{~m} \mathrm{~s}^{-2}, \ldots . ..\right) .+$, data at $3 \mathrm{~g}$ (from Bjurstedt et al. 1968); data at $1 \mathrm{~g}$ (present study, and from Bjurstedt et al. 1968, Michel $e$ al. 1977); $\square$, data at $0 \mathrm{~g}$ (present study, and from Michel et al. 1977).

postural work alone. In addition, the data by Bjurstedt et al. (1968) did not show any increase in the $\dot{V}_{\mathrm{O}_{2}}$ difference although the difference in ventilation between the $3 g$ and the $1 g$ condition became progressively larger. Using the $m_{\mathrm{L}}$ of subject 1 and assuming a constant $f_{\mathrm{p}}$ value of 60 r.p.m., we were able to plot (Fig. 3) the relationship between $\dot{V} \mathrm{O}_{2}$ and $\dot{w}$ for different values of $a_{\mathrm{g}}$ from Eqn 7. In Fig. 4, the experimental values measured by others (Rosenhamer 1968, Bjurstedt et al. 1968, Nunneley \& Shindell 1975) as well as in the present study are also reported. Differences in $f_{\mathrm{p}}$ and in $m_{\mathrm{L}}$ may account for the error between experimental and theoretical data. The results of Fig. 3 justify the contention that changes in $\dot{E}_{\text {int }}$ explain the $\dot{V}_{\mathrm{O}_{2}}$ differences at any given $\dot{w}$ between 0,1 and $3 \mathrm{~g}$. The results of this study do not allow refutation of the hypothesis that the $\dot{V}_{\mathrm{O}_{2}}$ during dynamic leg exercise on a cycle ergometer depends on $a_{\mathrm{g}}$.

The experiments described in this study were part of the EUROMIR 95 long-term space mission, jointly organized by the European Space Agency and the Russian Space Agency. The experiments on Earth were carried out at the European Astronaut Centre, Cologne, Germany. The experiments in microgravity were carried out in the Russian Space Station Mir. The study was supported by the European Space Agency, the Federal Swiss Prodex Fund (grant $n^{\circ} 142967$ ), the National Swedish Space Board of the Swedish Medical Research Council (grant $n^{\circ} 5020$ ), and the National Aeronautics and Space Administration, USA (grant $n^{\circ} \mathrm{NAGW}$ 3937). The European Space Agency also provided technical support to the experiment. The excellent collaboration obtained from the prime and back-up crew of EUROMIR 95 is acknowledged.

\section{REFERENCES}

Anton-Kuchly, B., Roger, P. \& Varène, P. 1984. Determinants of increased energy cost of submaximal exercise in obese subjects. J Appl Physiol 56, 18-23.
Åstrand, I., Åstrand, P.O. \& Stunkard, A. 1960. Oxygen intake of obese individuals during work on a bicycle ergometer. Acta Physiol Scand 50, 294-299.

Banister, E.W. \& Jackson, R.C. 1967. The effect of speed and load changes on oxygen intake for equivalent power output during bicycle ergometry. Int $Z$ Angew Physiol 24, 284-290.

Bjurstedt, H., Rosenhamer, G. \& Wigertz, O. 1968. High-G environment and response to graded exercise. J Appl Physiol 25, 713-719.

Camus, G., Atchou, G., Brückner, J.C., Giezendanner, D. \& di Prampero, P.E. 1988. Slow upward drift of $\dot{V} \mathrm{O}_{2}$ during constant-load cycling in untrained subjects. Eur J Appl Physiol 58, 197-202.

Cerretelli, P. \& di Prampero, P.E. 1987. Gas exchange in exercise. In: L.E. Farhi \& S.M. Tenney (eds) Handbook of Physiology. The Respiratory System. Gas Exchange, Sect. 3, Vol. IV, pp. 297-339. American Physiological Society, Bethesda MD, USA.

Convertino, V.A. 1996. Exercise and adaptation to microgravity environments. In: M.J. Fregly \& C.M. Blatteis (eds) Handbook of Physiology. Environmental Physiology, Vol. II, pp. 815-843. Oxford University Press, New York.

Cotes, J.E. 1969. Relationships of oxygen consumption, ventilation and cardiac frequency to body weight during standardized submaximal exercise in normal subjects. Ergonomics 12, 415-427.

Dickinson, S. 1929. The efficiency of bicycle pedalling as affected by speed and load. J Physiol Lond 67, 242-255.

di Prampero, P.E., Cortili, G., Mognoni, P. \& Saibene, F. 1979. Equation of motion of a cyclist. J Appl Physiol 47, 201-206.

Francescato, M.P., Girardis, M. \& di Prampero, P.E. 1995. Oxygen cost of internal work during cycling. Eur J Appl Physiol 72, 51-57.

Gaesser, G.A. \& Brooks, G.A. 1975. Muscular efficiency during steady-rate exercise: effects of speed and work rate. J Appl Physiol 38, 1132-1139.

Garry, R.C. \& Wishart, G.M. 1931. On the existence of a most efficient speed in bicycle pedalling, and the problem of determining human muscular efficiency. J Physiol Lond 72, 426-437.

Henry, F.M. \& De Moor, J. 1950. Metabolic efficiency of exercise in relation to work load at constant speed. J Appl Physiol 2, 481-487.

Kallay, M.C., Hyde, R.W. \& Smith, R.J. 1990. Assessment of rebreathing $\mathrm{O}_{2}$ consumption in humans with normal and diseased lungs. J Appl Physiol 68, 1443-1452.

Kamon, E., Metz, K.F. \& Pandolf, K.B. 1973. Climbing and cycling with additional weights on the extremities. J Appl Physiol 35, 367-370.

Kaneko, M. \& Yamazaki, T. 1978. Internal mechanical work due to velocity changes of the limb in working on a bicycle ergometer. In: E. Asmussen \& K. Jorgensen (eds) Biomechanics VI-A, pp. 86-92. University Park Press, Baltimore, MD, USA.

Kleinbaum, D.G., Kupper, L.L. \& Muller, K.E. 1988. Applied Regression Analysis and Other Multivariate Methods. PWS-Kent, Boston. 
Le Blanc, A., Rowe, R., Schneider, V., Evans, H. \& Hedrick, T. 1995. Regional muscle loss after short duration spaceflight. Aviat Space Environ Med 66, 1151-1154.

Levine, B.D., Lane, L.D., Watenpaugh, D.E., Gaffney, F.A., Buckey, J.C. \& Blomqvist, C.G. 1996. Maximal exercise performance after adaptation to microgravity. J Appl Physiol 81, 686-694.

Linnarsson, D. 1974. Dynamics of pulmonary gas exchange and heart rate changes at start and end of exercise. Acta Physiol Scand 425 (Suppl), 1-68.

Luhtanen, P., Rahkila, P., Rusko, H. \& Viitasalo, J.T. 1987. Mechanical work and efficiency in ergometer bicycling at aerobic and anaerobic thresholds. Acta Physiol Scand 131, 331-337.

Michel, E.L., Rummel, J.A., Sawin, C.F., Buderer, M.C. \& Lem, J.D. 1977. Results of Skylab medical experiment M171. Metabolic activity. In: S. Johnson \& L.F. Dietlein (eds) Biomedical Results from Skylab, pp. 372-387. National Aeronautics and Space Administration, Washington, DC.

Nunneley, S.A. \& Shindell, D.S. 1975. Cardiopulmonary effects of combined exercise and $+\mathrm{Gz}$ acceleration. Aviat Space Environ Med 46, 878-882.

Otis, A.B. 1964. Quantitative relationship in steady-state gas exchange. In: W.O. Fenn \& H. Rahn (eds) Handbook of Physiology. Respiration, Sect. 3, Vol. I, pp. 681-698. American Physiological Society, Washington, DC.
Pendergast, R.D., Olszowka, A.J., Rokitka, M.A. \& Farhi, L.E. 1987. Gravitational force and cardiovascular system. In: P. Dejours (ed.) Comparative Physiology of Environmental Adaptations, pp. 15-26. Karger, Basel, Switzerland.

Prisk, G.K., Elliott, A.R., Guy, H.J.B., Kosonen, J.M. \& West, J.B. 1995. Pulmonary gas exchange and its determinants during sustained microgravity on Spacelabs SLS-1 and SLS-2. J Appl Physiol 79, 1290-1298.

Rosenhamer, G. 1968. Influence of increased gravitational stress on the adaptation of cardiovascular and pulmonary function to exercise. Acta Physiol Scand 276 (Suppl), 1-61.

Wells, R., Morrissey, M. \& Hughson, R. 1986. Internal work and physiological responses during concentric and eccentric cycle ergometry. Eur J Appl Physiol 55, 295-301.

Whipp, B.J. \& Wasserman, K. 1969. Efficiency of muscular work. J Appl Physiol 26, 644-648.

Whipp, B.J. \& Wasserman, K. 1972. Oxygen uptake kinetics for various intensities of constant-load work. J Appl Physiol 33, 351-356.

Widrick, J.J., Freedson, P.S. \& Hamill, J. 1992. Effect of internal work on the calculation of optimal pedalling rates. Med Sci Sports Exerc 24, 376-382.

Winter, D.A. 1979. Biomechanics of Human Movement. Wiley, New York. 